\title{
Damage detection in stiffened plates by wavelet transform
}

\author{
Joe-Ming Yang, Zen-Wei Yang and Chien-Ming Tseng
}

Department of Systems and Naval Mechatronic Engineering, National Cheng Kung University, Tainan, Taiwan, ROC

\begin{abstract}
In this study, numerical analysis was carried out by using the finite element method to construct the first mode shape of damaged stiffened plates, and the damage locations were detected with two-dimensional discrete wavelet analysis. In the experimental analysis, four different damaged stiffened structures were observed. Firstly, each damaged structure was hit with a shaker, and then accelerometers were used to measure the vibration responses. Secondly, the first mode shape of each structure was obtained by using the wavelet packet, and the location of cracks were also determined by two-dimensional discrete wavelet analysis. The results of the numerical analysis and experimental investigation reveal that the proposed method is applicable to detect single crack or multi-cracks of a stiffened structure. The experimental results also show that fewer measurement points are required with the proposed technique in comparison to those presented in previous studies.
\end{abstract}

KEY WORDS: Wavelet Packet; Two-dimensional Discrete Wavelet Analysis; Damage Detection; Stiffened Structure.

\section{INTRODUCTION}

During recent decades, wavelet analysis has been an useful tool in detecting damage in structures. However, most researches have focused on the damage identification in basic structures such as beams, plates and bearings. In practical application, the basic structures do not exist alone. Instead, they are usually accompanied with one another, and these composite structures are commonly used in ships. Wavelet transform can resolve time domain-frequency domain problems more effectively and has a great capability in reconstructing the decomposed signal. In this study, a method for crack identification in stiffened plates based on twodimensional wavelet analysis was investigated.

French physicist Morlet $(1982,1983)$ determined that the Fourier transform was unable to analyze seismic waves during short time periods, so Goupillaud, Grossmann and Morlet (1984) later applied the wavelet concept on this signal analysis technique. Afterwards, Meyer (1986) and Mallat (1988) introduced the multi-resolution concept into wavelet analysis to form discrete wavelet transform.

Cawley and Adams (1979) combined measurements of natural frequencies from the experimental results and the finite element analysis to detect damage locations in structures. Daubechies (1992) constructed the orthogonal and compact support wavelet, which had local resolution in both time and frequency domains. Surace and Ruotolo (1994)

Corresponding author: Joe Ming Yang

e-mail: z7908036@email.ncku.edu.tw simulated the vibration signal of a cracked beam by wavelet transform. Furthermore, Wang and Mcfadden (1996) handled the vibration signal of a gear box with wavelet transform. Rucka and Wilde (2006) analyzed the modal shape of a cracked cantilever beam and plate by utilizing continuous wavelet transform to identify the damaged area. Yang and Hwang (2008) carried out a method involving both wavelet packet node norm and two-dimensional discrete wavelet transform (DWT2) which, in return, measured the first mode shape of aluminum alloy plates and found the location of cracks. In this paper, the first modal shape for an aluminum rectangular plate with stiffeners was obtained by using wavelet packet node norm initially, and then the damage locations were detected by two-dimensional discrete wavelet transform.

\section{WAVELET TRANSFORM}

Wavelet transform is widely used to resolve time domainfrequency domain problems. The basis of a wavelet transform is composed of translation and dilation functions. These functions are orthogonal functions and are formulated by shifting and expanding a basic wavelet function according to high or low frequency signals, which are to be analyzed. With the above features, the wavelet transform has great capability to display high time domain resolution at high frequencies and high frequency domain resolution at low frequencies. 


\section{Continuous wavelet transform}

In one-dimensional space, for a signal $f(t) \in L^{2}(R)$ with $-\infty \leq t \leq \infty$ continuous wavelet transform (CWT) is the convolution of the signal function with the wavelet function (mother wavelet) $\psi(t) \in L^{2}(R)$, and can be expressed as:

$W f(s, u)=\frac{1}{\sqrt{s}} \int_{-\infty}^{\infty} f(t) \bar{\psi}\left(\frac{t-u}{s}\right) d t$

Where $\bar{\psi}(t)$ is the complex conjugate of $\psi(t)$, and the variables $s$ and $u$ represent the scale and translation parameters, respectively. As the variable $s$ is increased, the period of the wavelet is also increased and vice versa. Therefore, the variable $s$ is considered as a trade-off variable between the time domain resolution and the frequency domain resolution. The term $\frac{1}{\sqrt{s}} \bar{\psi}\left(\frac{t-u}{s}\right)$ is the wavelet basic function (baby wavelet), and $1 / \sqrt{s}$ is a factor which allows the energy to remain the same before and after the transformation.

\section{Discrete wavelet transform}

The CWT method transforms a one-dimensional single variable function into a two-dimensional double variable function as described in the section above. In this transformation, a lot of redundancy and computational complexities are produced. To reduce the computing time and to save the memory space, scientists developed the discrete wavelet transform (DWT) based on the discrete scale parameter $s$ and the translation parameter $u$. The decomposition and reconstruction of DWT adopt the Mallat algorithm.

The scaling function $\phi$ associates with one-dimensional multi-resolution approximation $\left\{V_{j}\right\}_{j \in Z}$ where $j$ corresponds to the scale level as a scaling function. In two-dimensional discrete wavelet analysis, the approximation space $\left\{V_{j}^{2}\right\}_{j \in Z}$ is the separable two-dimensional multi-resolution and is defined as $V_{j}^{2}=V_{j} \otimes V_{j}$ where $\otimes$ denotes the tensor product, and $V_{j}$ represents the one-dimensional space. Considering $W_{j}^{2}$ as the detail space, which is the orthogonal complement of the lower resolution approximation space $V_{j}^{2}$ in $V_{j-1}^{2}, V_{j-1}^{2}$ can be expressed as

$V_{j-1}^{2}=V_{j}^{2} \oplus W_{j}^{2}$

Where $\oplus$ denotes the direct sum of two orthogonal vector spaces. Based on Equation (2), a wavelet orthogonal basis on $L^{2}\left(R^{2}\right)$ can be constructed by using the scaling function $\varphi(x)$ and the corresponding wavelet $\psi(x)$. The wavelet $\psi(x)$ is the one-dimensional wavelet associated with the scaling function $\varphi(x)$. Details of the derived twodimensional discrete wavelet transform can be found in Mallat et al. (1988). In particular, the jointed scaling function and three wavelets are defined as follows:

$\phi(x, y)=\phi(x) \phi(y)$

$\Psi^{1}(x, y)=\phi(x) \Psi(y)$

$\Psi^{2}(x, y)=\Psi(x) \phi(y)$

$\Psi^{3}(x, y)=\Psi(x) \Psi(y)$

The approximation signal is defined as $\phi(x, y)$ and $\psi^{1}(x, y)$, $\psi^{2}(x, y)$ and $\psi^{3}(x, y)$ denote the signals in the horizontal, vertical and diagonal directions, respectively. One easier method in proceeding with DWT2 is to employ either a certain wavelet or particular wavelet decomposition filters (Lo_D and Hi_D). Fig. 1 shows the basic decomposition steps for DWT2 where $\mathrm{CA}_{\mathrm{j+1}}$ is the approximate signal, and the notations $\mathrm{CD}_{\mathrm{j}+1}^{\mathrm{h}}, \mathrm{CD}_{\mathrm{j}+1}^{\mathrm{V}}$ and $\mathrm{CD}_{\mathrm{j}+1}^{\mathrm{d}}$ are the detail signals in the horizontal, vertical, and diagonal orientations, respectively.

In previous studies (Yang, 2008), it was established that the 2-D DWT method could detect small disturbances in the plate, and the diagonal detailed signal could pinpoint the locations of cracks in an alloyed plate. Hence, 2-D DWT was carried out in this study to locate cracks or damaged areas.

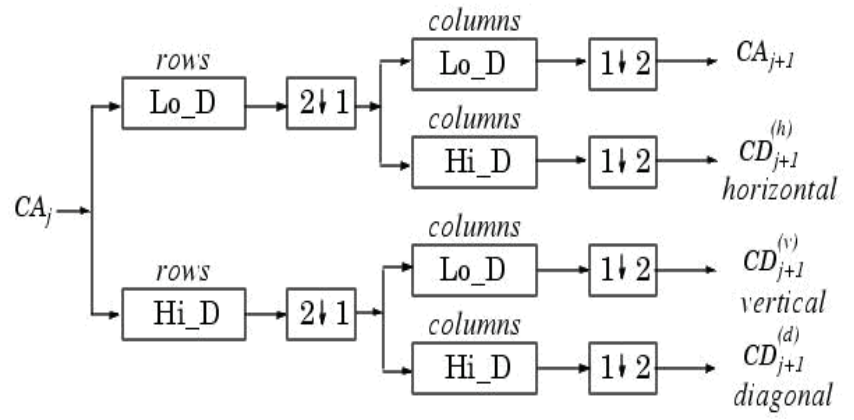

Fig. 1 Decomposition steps for DWT2.

\section{Wavelet packet decomposition}

The wavelet packet method is a generalization of wavelet decomposition that offers a richer signal analysis. The wavelet packet is waveforms indexed by three naturally interpretable parameters: position, scale (as in wavelet decomposition) and frequency. For a given orthogonal wavelet function, a library known as the wavelet packet base has the exact features. Each of these bases provides a particular way of coding signals to preserve global energy and to reconstruct the same features. 
The wavelet packets can be applied to numerous expansions of a given signal. The most suitable decomposition of a set signal with respect to an entropy-based criterion is selected. There exist simple and efficient algorithms for both wavelet packet decomposition and optimal decomposition. The adaptive filtering algorithm with direct application in optimal signal coding and data compression is then produced. As shown in Fig. 2, the wavelet packet transform can be viewed as a tree diagram. The root of the tree is the original data set. After the first transform step, the low pass result is located on the left branch of the tree diagram, and the high pass result is positioned on the right branch. The second level of the tree diagram consists of results from the next step of the wavelet transform. Subsequent levels in the tree are created by recursively applying the wavelet transform step to the previous low and high pass filter results.

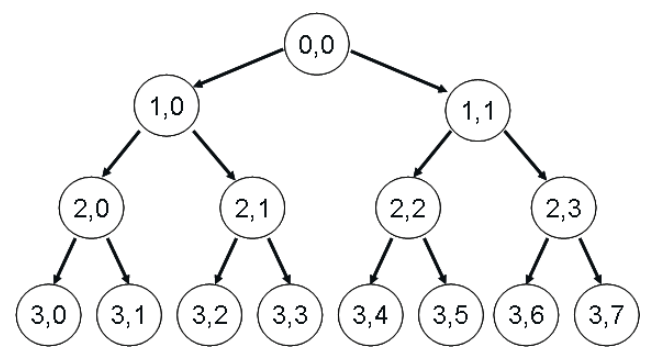

Fig. 2 Wavelet packet tree.

\section{Wavelet packet node norm}

Wavelet packet coefficient is the inner product of the signal function $f(t)$ and the wavelet packet function $W_{j, n, k}(t)$ :

$w_{j, n, k}=\left\langle f, W_{j, n, k}\right\rangle=\int f(t) W_{j, n, k}(t) d t$

The wavelet packet node energy, which has its own special physical characteristics, presents a more powerful identification ability than the wavelet packet coefficients.

$c_{j, n} \equiv \sum_{k} w_{j, n, k}^{2}$

Let $C_{j, n}$ denotes the energy in the frequency band at the same wavelet packet node $(j, n)$, and then the wavelet packet node norm can be expressed as the square root of the wavelet packet node energy:

$\left\|w_{j, n}\right\|_{2}=\left(c_{j, n}\right)^{1 / 2}$

In the experiment analysis, the wavelet packet node norm was calculated for each measured point.

\section{NUMERICAL SIMULATION BY ANSYS}

Before applying the wavelet transform to experimental analysis, the numerical simulations were performed at first. A plate of dimensions $54 \mathrm{~cm} \times 30 \mathrm{~cm} \times 0.3 \mathrm{~cm}$ and four stiffeners of dimensions $54 \mathrm{~cm} \times 3 \mathrm{~cm} \times 0.3 \mathrm{~cm}$ and $30 \mathrm{~cm} \times 3 \mathrm{~cm} \times 0.3 \mathrm{~cm}$ were utilized to form the 3-D finite model shown in Fig. 3. The distance between the stiffeners and the edge of the plate was $3 \mathrm{~cm}$. Each mesh, which was in the plate, was of $1 \mathrm{~cm} \times$ $1 \mathrm{~cm}$, where plate was divided into 1620 meshes. The damaged area had dimensions of $3 \mathrm{~cm} \times 3 \mathrm{~cm} \times 0.1 \mathrm{~cm}$ is shown in Fig. 3. The first mode shape of the plate is shown in Fig. 4.

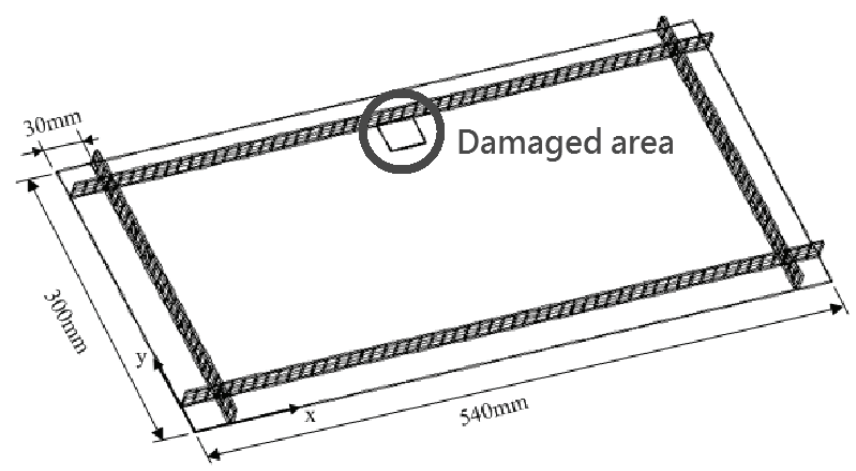

Fig. 3 Schematic diagram of the stiffened plate.

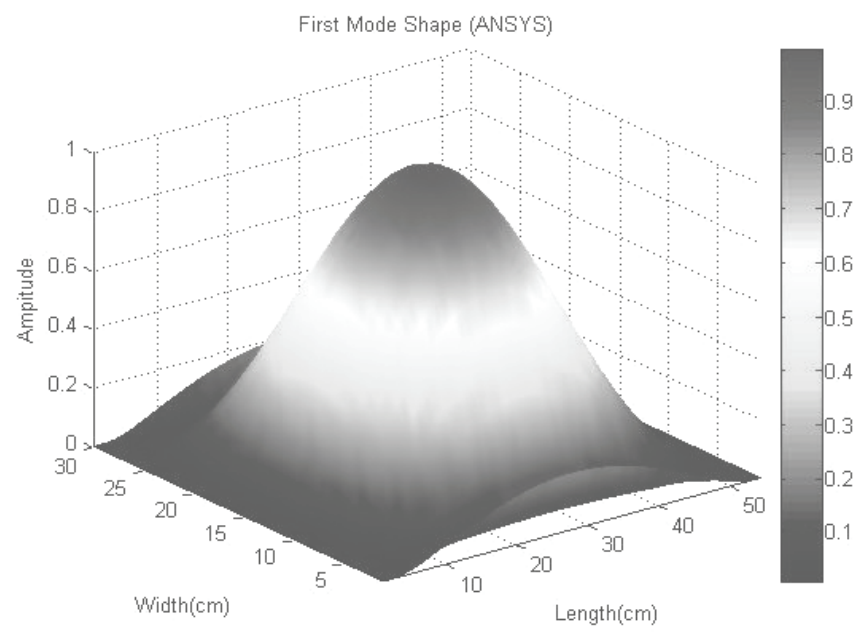

Fig. 4 First mode shape of the stiffened plate.

In the case of two-dimensional signals, the space-scale representation of a signal is a three-dimensional problem. The result of the two-dimensional wavelet transform of the plate mode shape has four wavelet coefficients as shown in Fig. 1 : The first one is the approximate signal $\mathrm{CA}_{1}$, the others are three detailed signals, including the horizontal detailed signal $\mathrm{CD}_{1}{ }^{1}$, the vertical detailed signal $\mathrm{CD}_{1}{ }^{2}$ and the diagonal detailed signal $\mathrm{CD}_{1}{ }^{3}$. In this investigation, we chose Symlets 4 wavelet to operate two-dimensional discrete wavelet analysis. Furthermore the effects of other types of wavelet are also discussed in the following section. The approximate signal $\mathrm{CA}_{1}$ in Fig. 5(a) indicates the smoothed version of the mode of vibration for the damaged plate. Fig. 
5(b) displays the horizontal detailed signal $\mathrm{CD}_{1}{ }^{1}$. The vertical detailed signal $\mathrm{CD}_{1}{ }^{2}$ and the diagonal detailed signal $\mathrm{CD}_{1}{ }^{3}$ are shown in Fig. 5(c) and Fig. 5(d), respectively. Only the vertical detailed signal $\mathrm{CD}_{1}{ }^{2}$ and the diagonal detailed signal $\mathrm{CD}_{1}{ }^{3}$ can identify the approximate location of the damage. It is found that the horizontal detailed signal $\mathrm{CD}_{1}{ }^{1}$ is sensitive to the defects parallel to the $\mathrm{x}$ axis and the vertical detailed

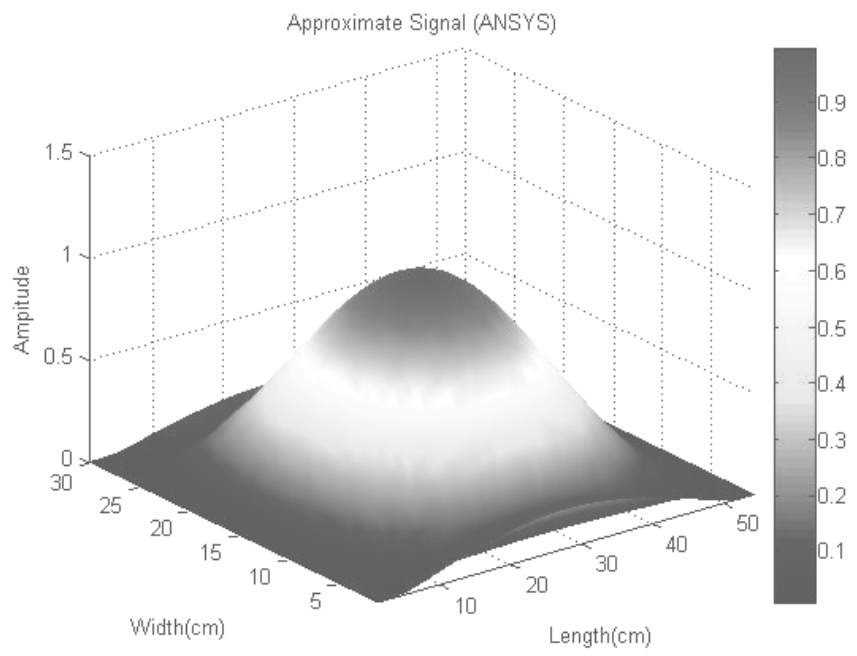

(a) Approximate signal.

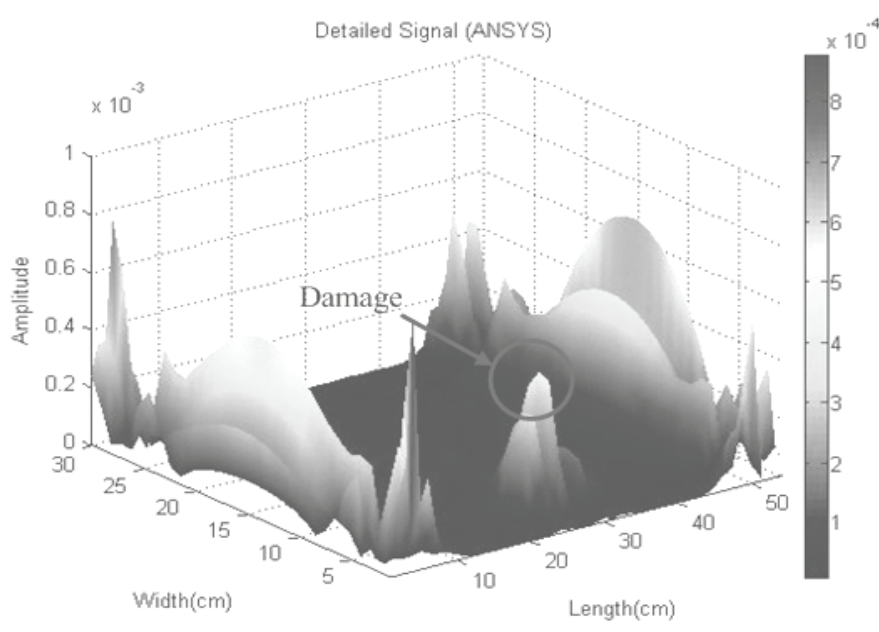

(c) Vertical detailed signal. signal $\mathrm{CD}_{1}{ }^{2}$ is sensitive to the defects parallel to the $\mathrm{y}$ axis. The diagonal detailed signal $\mathrm{CD}_{1}{ }^{3}$ is sensitive to the defects parallel to either the $\mathrm{x}$ or $\mathrm{y}$ orientation. The above results suggest that the diagonal detailed signal $\mathrm{CD}_{1}{ }^{3}$ should be considered in the present case. In the subsequent analysis, the diagonal detailed signal $\mathrm{CD}_{1}{ }^{3}$ is more suitable to be used for finding cracks of the stiffened plate.

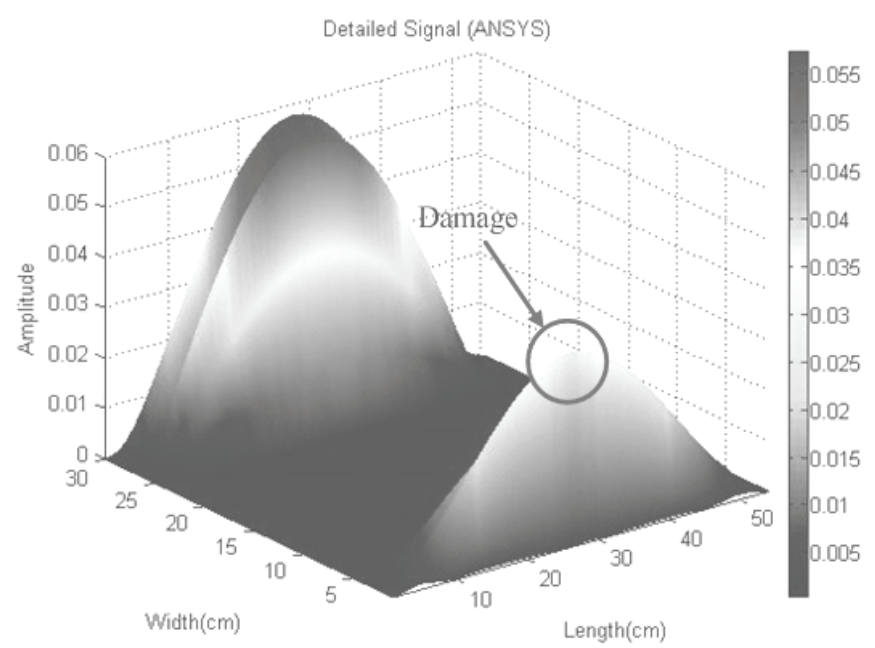

(b) Horizontal detailed signal.

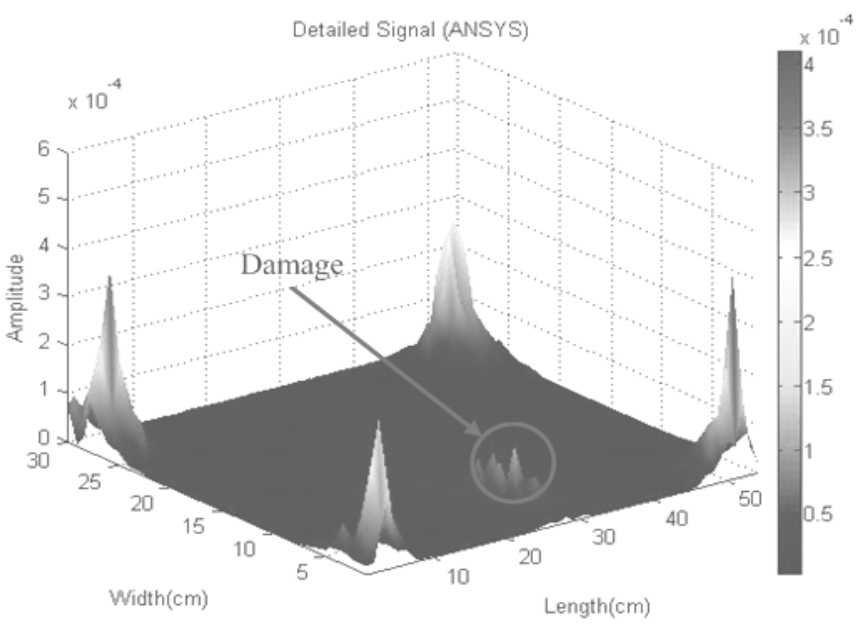

(d) Diagonal detailed signal.

Fig. 5 The signals after applying 2-D discrete wavelet transform on damaged plate of first modal shape.

Based on the discussion above, the damaged location or the crack location on a stiffened plate can be found by the two-dimensional discrete wavelet transform method. For the following scenario, the damaged area was simulated on the reverse side of the plate, and therefore, the location of the damage could not be seen directly on the main face of the plate. Nevertheless, the damaged area could be identified using 2-D discrete wavelet transform analysis. However, a problem arose as the peak signals caused by the damaged area was not distinct enough in Fig. 5(d) due to the interference of the stiffeners. This interference raised the amplitude of these signals compared to the signals produced just by the damaged area alone. Consequently, the analyzed area must be minimized in order to eliminate the effects caused by the stiffeners, and the measurement area was thus reduced to the shaded area shown in Fig. 6. The diagonal detailed signal of the shaded area is presented in Fig. 7. After we minimized the analyzed area, the location of the damaged area can be determined easily. 
In order to make comparisons with the experimental modal analysis, we simulated four different damaged stiffened plates by using ANSYS. The dimensions $4 \mathrm{~cm}$ $\times 1 \mathrm{~cm} \times 0.03 \mathrm{~cm}$ of the damaged area were selected. Case 1 ,

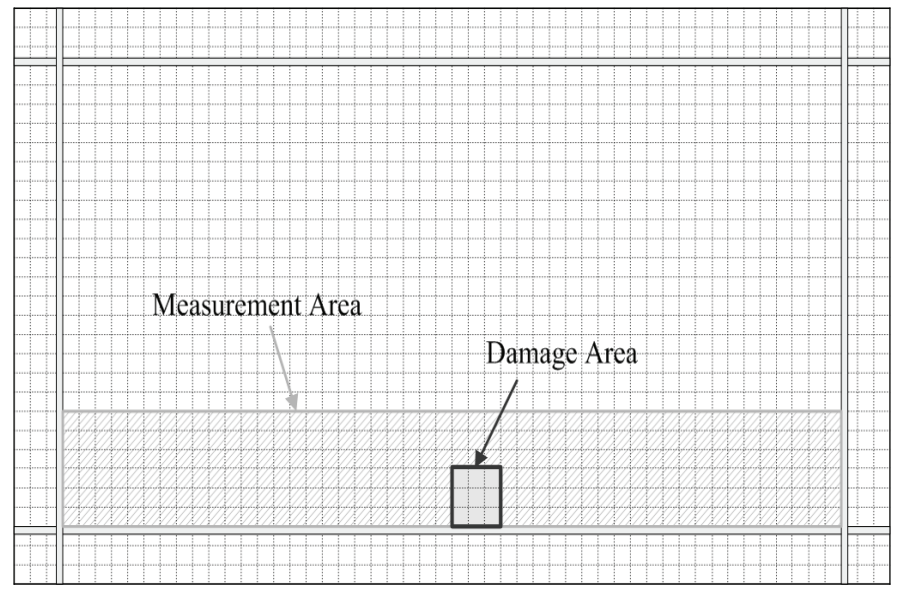

Fig. 6 Diminishing the analyzed area (shadow area).

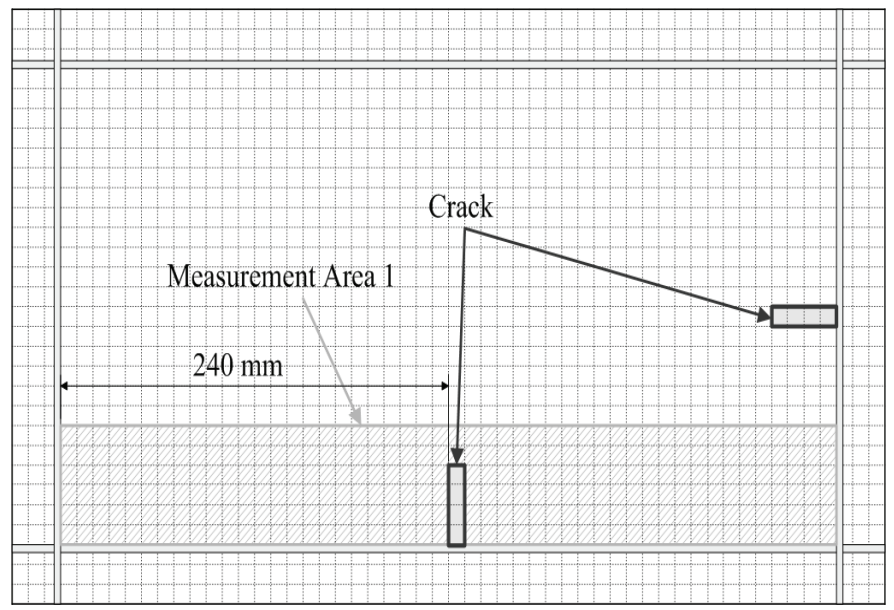

Fig. 8(a) Case1 (measurement area 1).

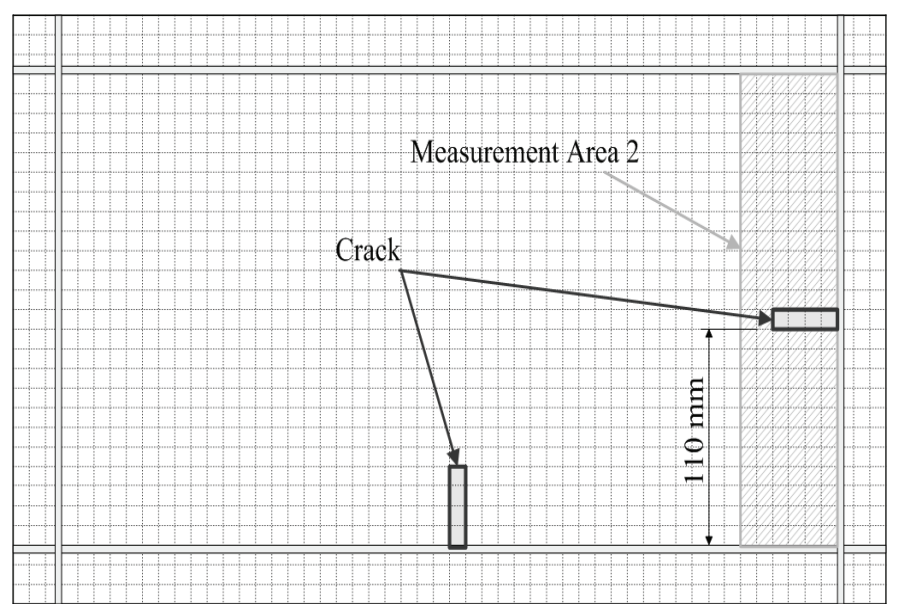

Fig. 9 (a) Case 1 (measurement area 2).

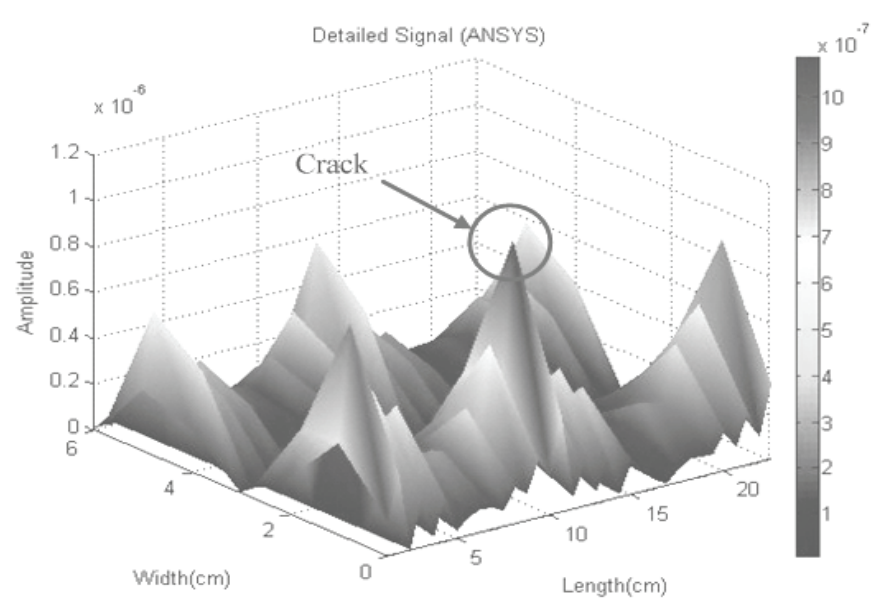

which has two measurement areas, is shown in Fig. 8(a) and Fig. 9(a), respectively. The diagonal detailed signals for measurement areas 1 and 2 are displayed in Fig. 8(b) and Fig. 9(b).

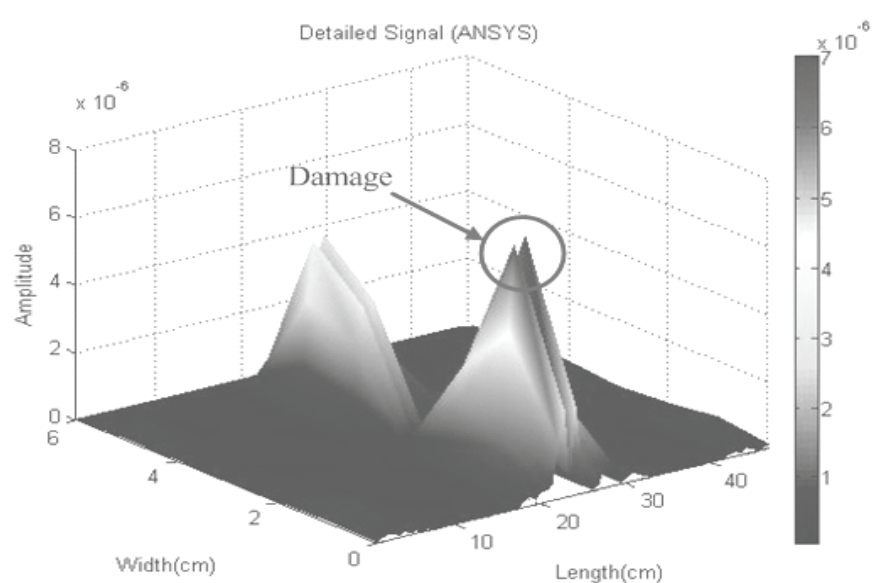

Fig. 7 Diagonal detailed signal of the shadowed area.

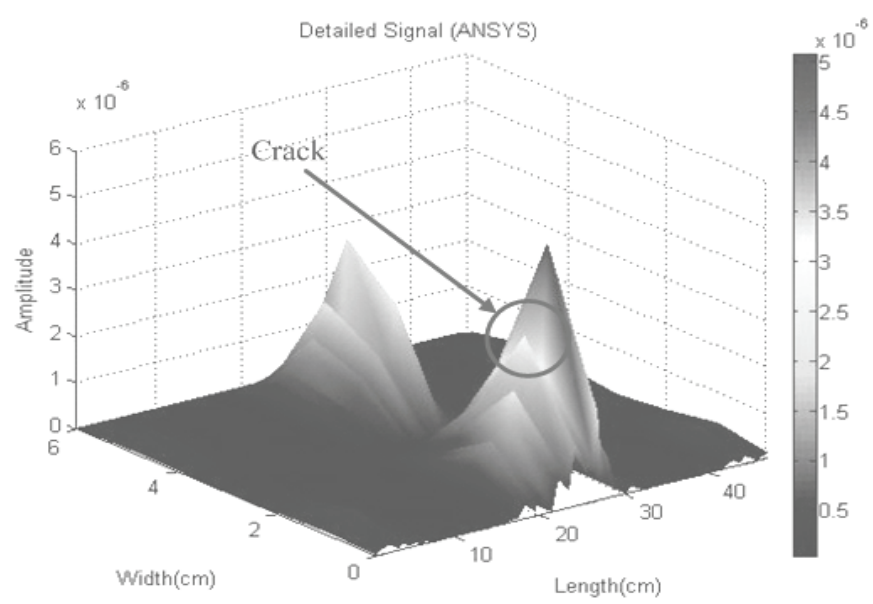

Fig. 8 (b) Diagonal detailed signal of measurement area1.

Fig. 9 (b) Diagonal detailed signal of measurement area2. 


\section{Comparisons of analysis using different types of wavelet}

It is important to choose the proper wavelet in order to operate wavelet transform. Fig. 8(b) shows the diagonal detailed signal based on Symlet 4 wavelet, and the crack can be identified in case 1 (measurement area 1). Moreover, four wavelet transform analysis depending on the different wavelet used are also discussed. Fig. 10 shows diagonal detailed signals based on Symlet 2, Symlet 5, Daubechy 5 and Coiflet 3. Ultimately, Symlet 4 is determined to be the best wavelet for detecting cracks on stiffened plates and is used in the following sections.

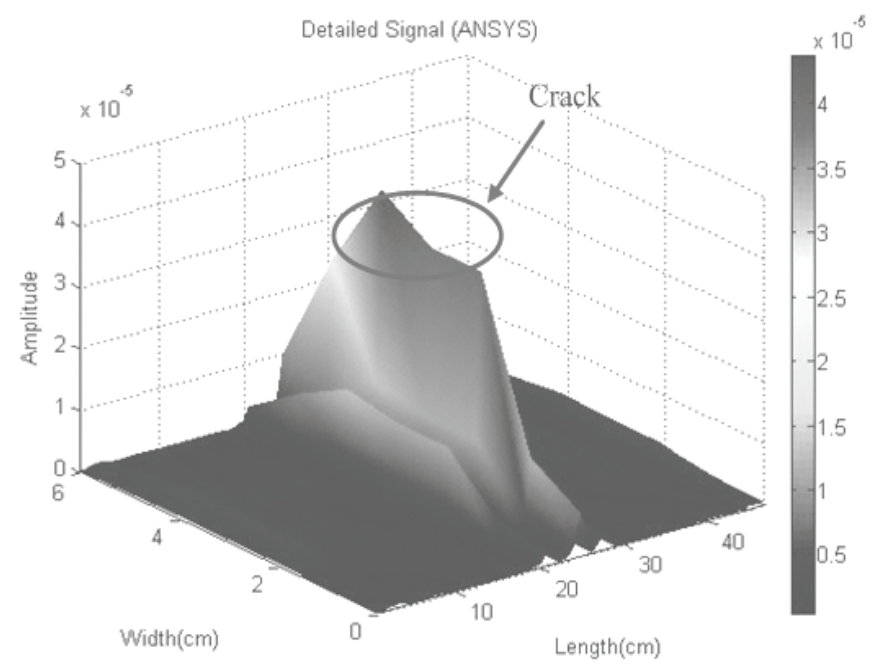

(a) Diagonal signal based on Symlet 2 wavelet.

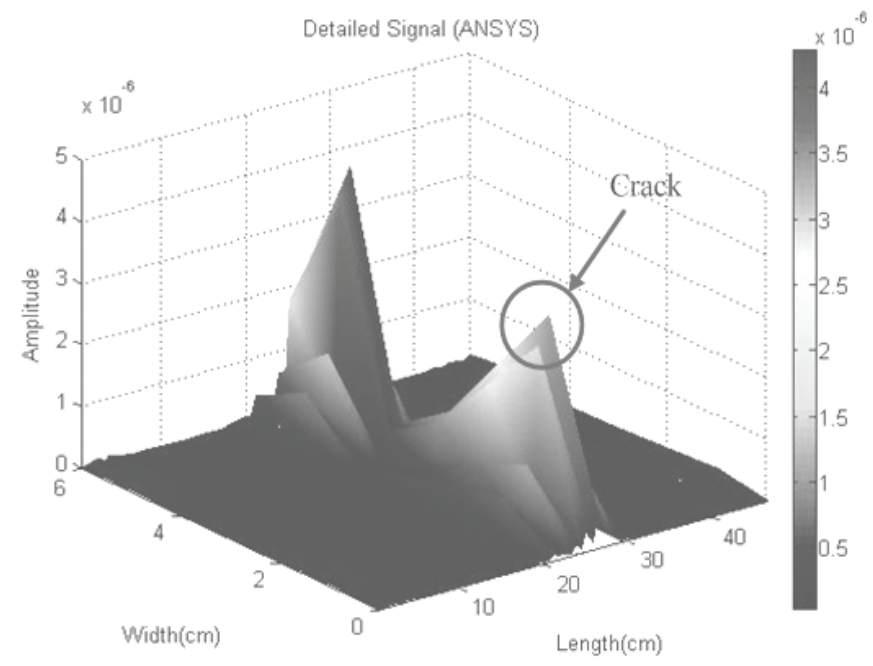

(c) Diagonal signal based on Daubechy 5 wavelet.

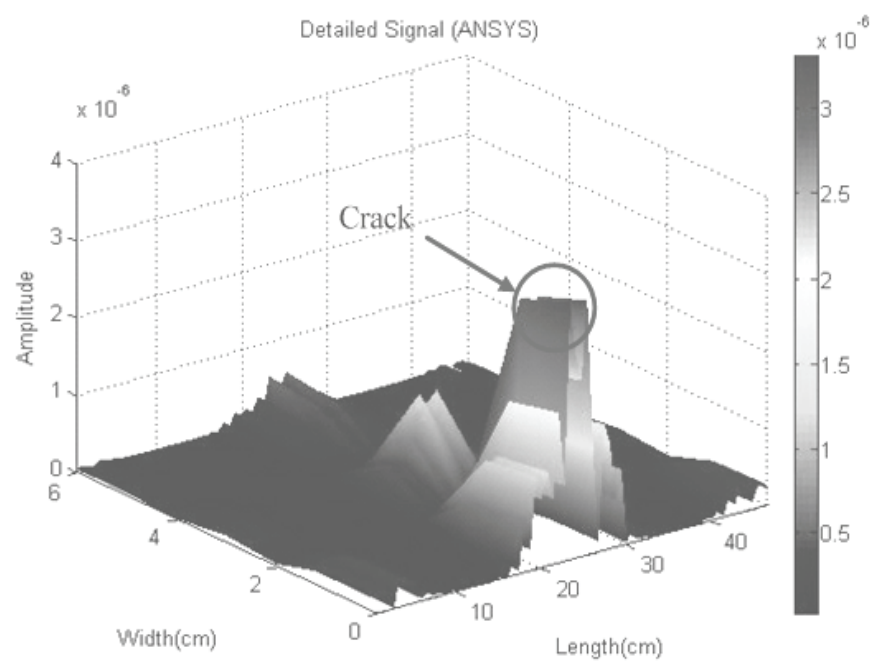

(b) Diagonal signal based on Symlet 5 wavelet.

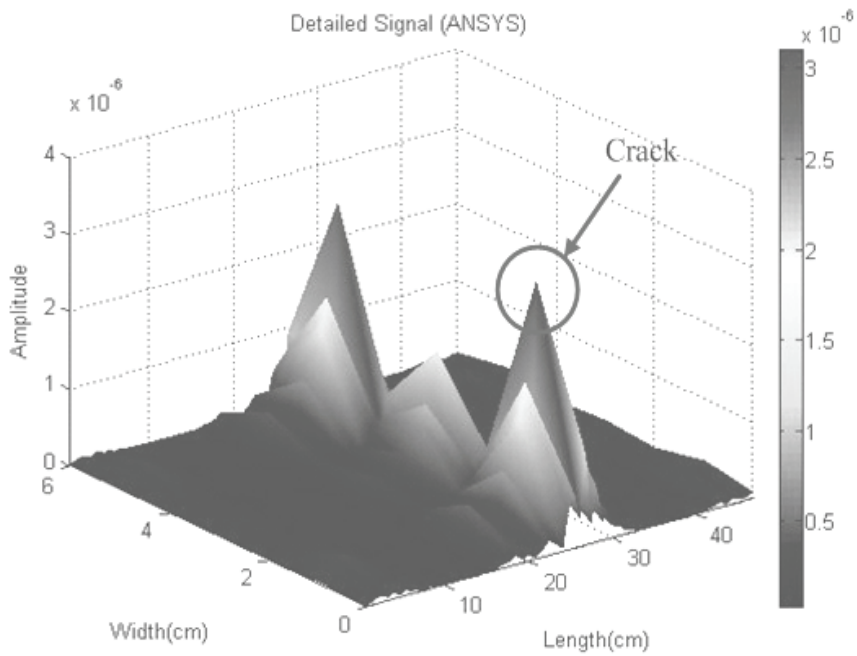

(d) Diagonal signal based on Coiflet 3 wavelet.

Fig. 10 Two-dimensional discrete wavelet transform based on four different wavelets.

\section{Identification of crack using Symlet 4 wavelet for stiffened plate}

The crack locations were chosen based on the amplitudes of the diagonal detailed signals. The amplitudes were affected by boundary conditions or the use of welding methods, and as a result, the relative peaks may be treated as possible crack locations. It is easy to identify the location of the damaged area shown in Fig. 8(b); however, it is not applicable to the case displayed in Fig. 9(b) due to the numerous noise signals found near the edges of the plate. These noises affect the determination of the damaged area and may be formed from the fixed end. Similar phenomena can be found in our experimental results. 


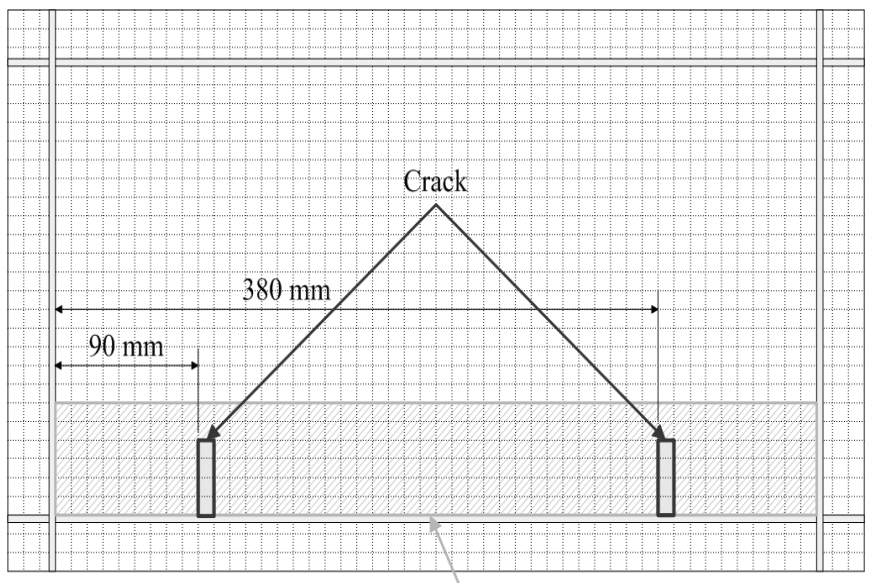

Measurement Area

(a) Measurement area of case 2 .

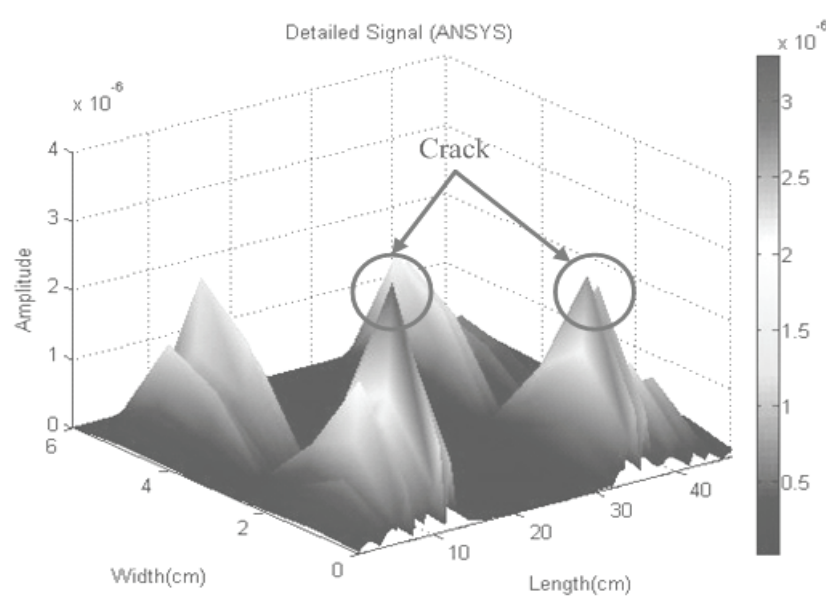

(b) Diagonal detailed signal of measurement area.

Fig. 11. (a) The measurement area of case 2 and (b) Diagonal detailed signal after 2-D DWT.

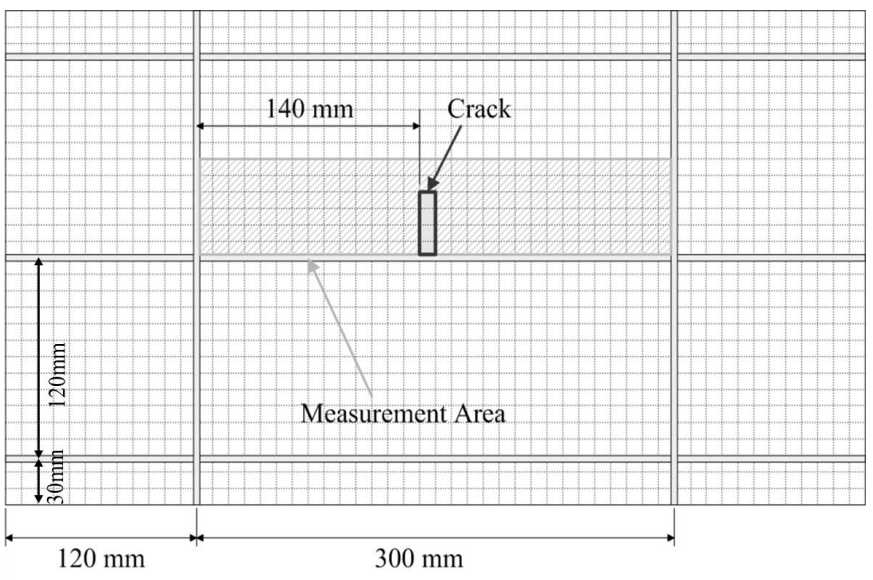

(a) Measurement area of case 3 .

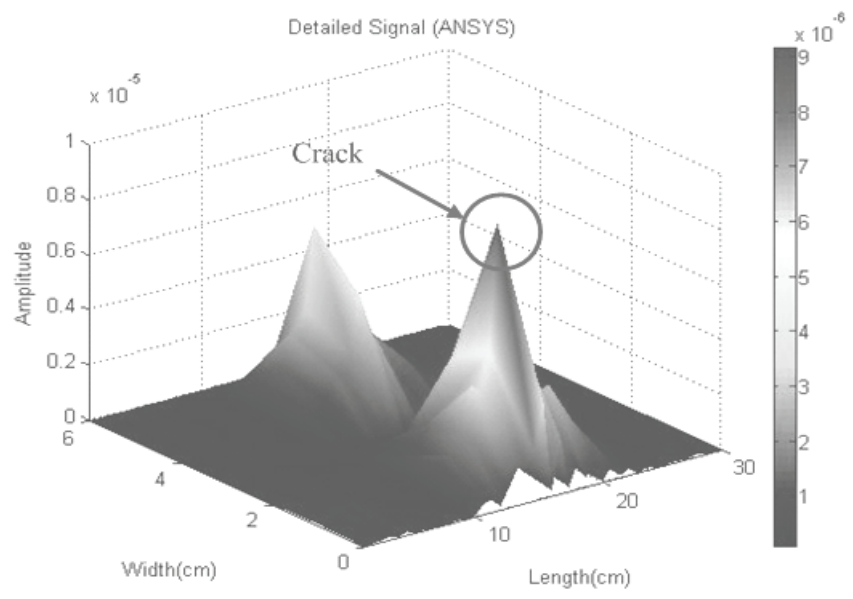

(b) Diagonal detailed signal of measurement area.

Fig. 12. (a) The measurement area of case 3 and (b) Diagonal detailed signal after 2-D DWT.

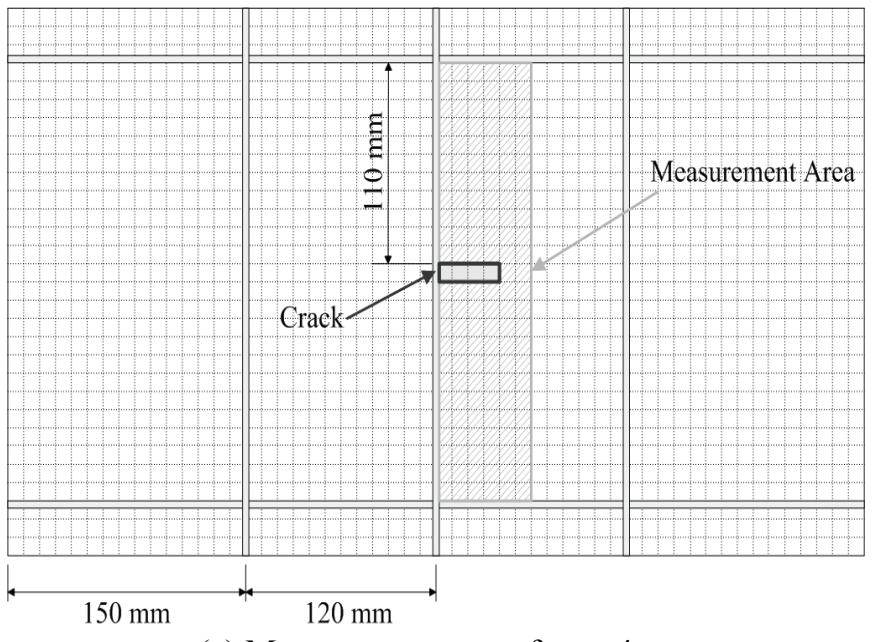

(a) Measurement area of case 4 .

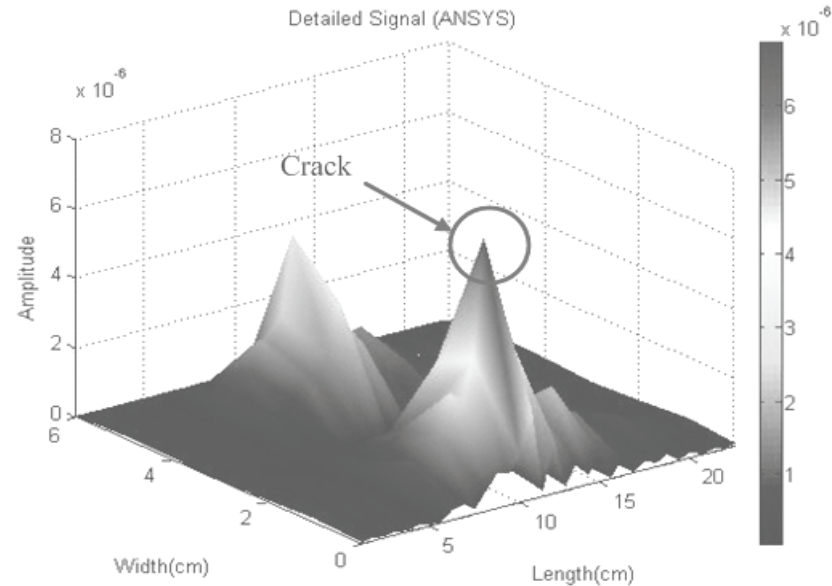

(b) Diagonal detailed signal of measurement area.

Fig. 13 (a) The measurement area of case 4 and (b) Diagonal detailed signal after 2-D DWT. 
Fig. 11(a) to 13(a) present three different measurement areas for various crack locations. The diagonal detailed signals which are obtained from the shaded area are presented in Fig. 11(b)-13(b), respectively. By comparing cases 1 and 3, we conclude that the simulation results are not affected by the free end. By distinguishing between case 1 and case 4 , the results reveal that the fixed end will produce a great amount of noise signals near the edges of the diagonal signal diagram, and these noise signals will affect the precision of determining the crack locations.

\section{EXPERIMENTAL MODAL ANALYSIS}

\section{Experimental procedure}

Firstly, a pulse signal generated by the program Labview was amplified and was then sent to the shaker to excite the stiffened plates. Afterwards, the responses of the stiffened plates were measured by accelerometers that were pasted onto the stiffened plates by using beeswax. Next, the measured waveforms were converted into digital values that could be recorded by computer. Fig. 14 portrays the above experimental procedure of signal analysis for the stiffened plates.

Each stiffened plate was composed of 6061T6 aluminum alloy. The length, width and thickness of the stiffeners and the plate were the same as the simulated ones. Young's modulus (E) and Poisson's ratio $(v)$ of the aluminum alloy material were $70000 \mathrm{Mpa}$ and 0.33 , respectively, and the density $(\rho)$ was $2700 \mathrm{Kg} / \mathrm{m}^{3}$. The crack's length was of $45 \mathrm{~mm}$, and the width of the crack was $1 \mathrm{~mm}$.

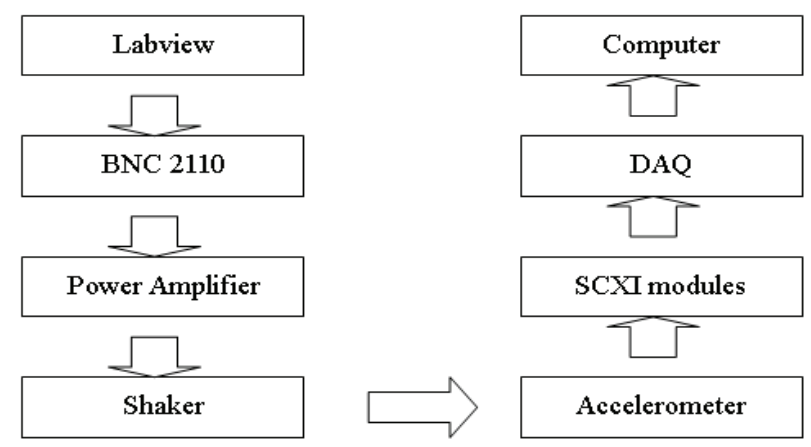

Fig. 14 Test facility flow chart.

Fig. 15 gives the top view of the test facilities and the boundary conditions of the stiffened plate. Case 1 has two measurements, as shown in Fig. 15 and Fig. 16. Fig. 15 to Fig. 19 display the arrangement of boundary conditions of the stiffened plates and the locations of the cracks for case 1 to case 4 , respectively. In the experiments, one accelerometer was used as the reference input whereas the second accelerometer was moved along the serial numbers located in the shaded area. The distance between one measured point to another was $2 \mathrm{~cm}$. In order to smoothen the transition from one point to another, an interpolation procedure was applied.

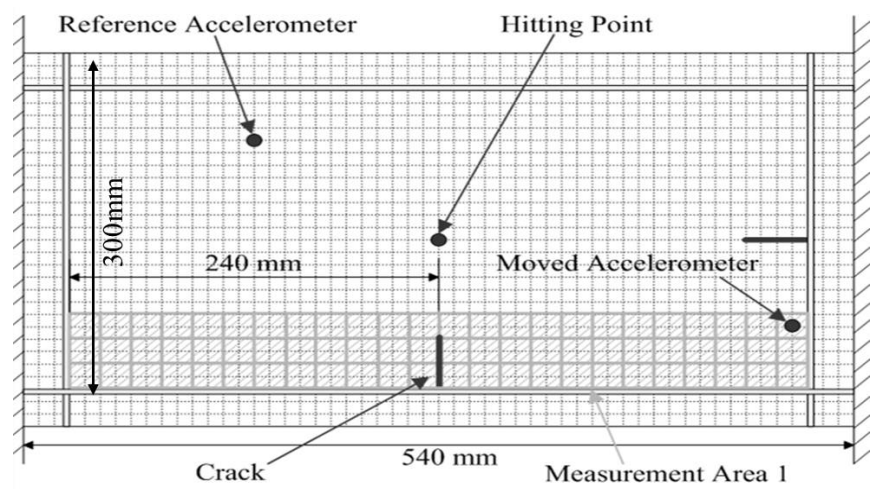

Fig. 15 Top view of case1(measurement area1).

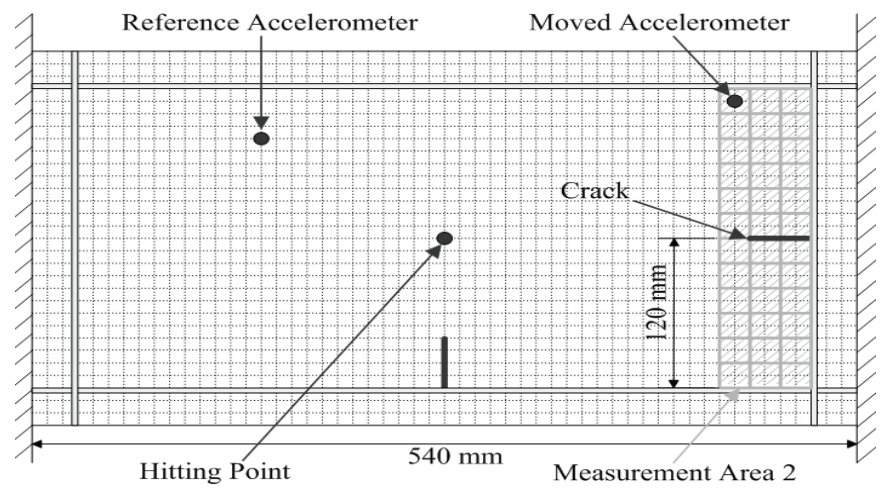

Fig. 16 Top view of Case1(measurement area 2).

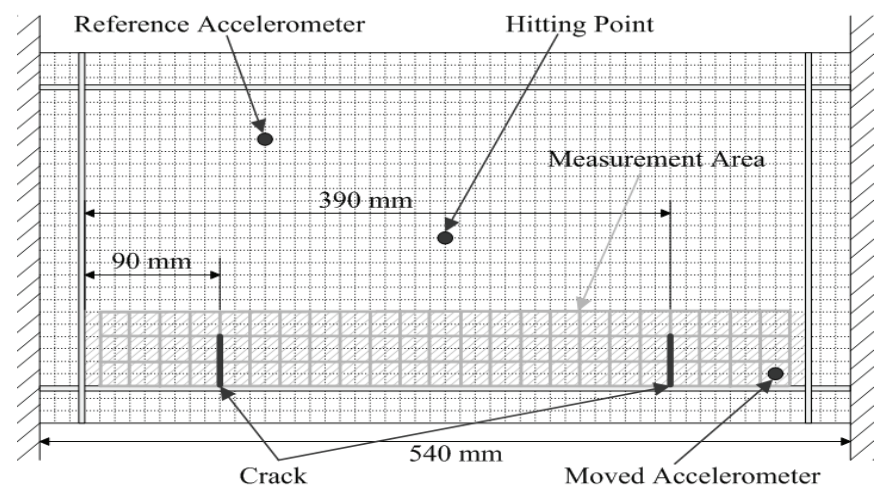

Fig. 17 Case 2.

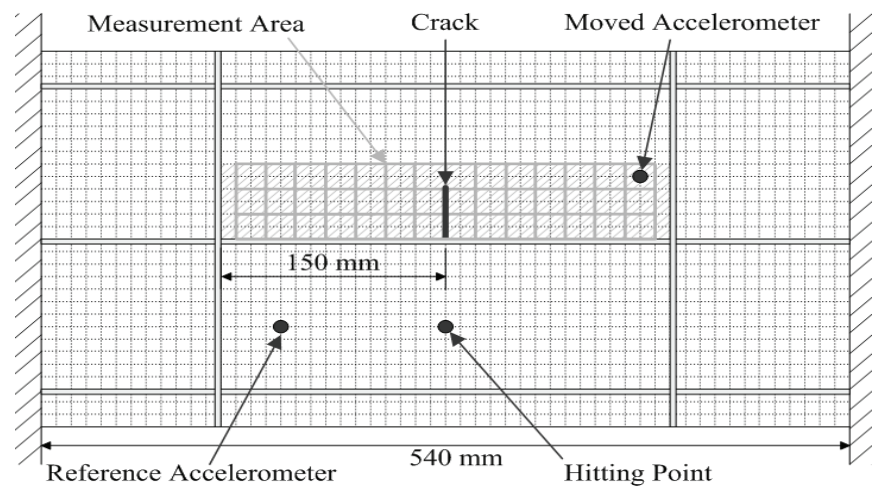

Fig. 18 Case3. 


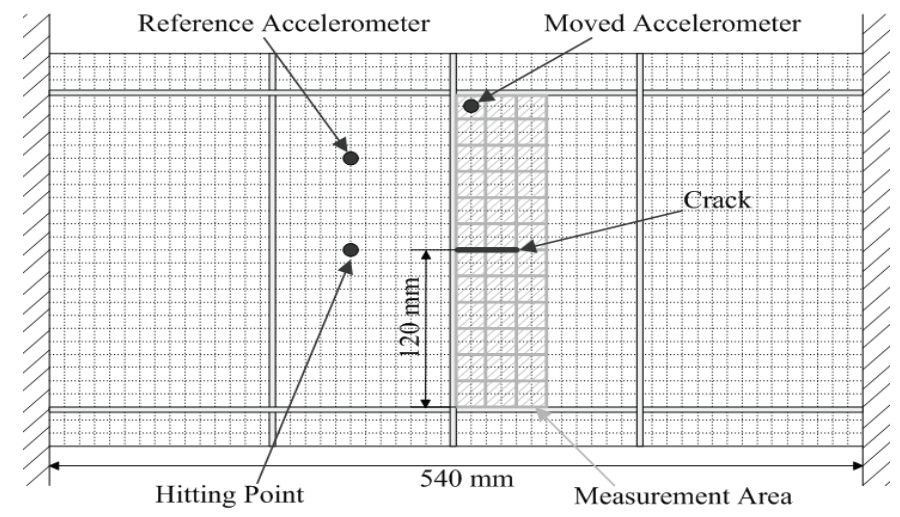

Fig. 19 Case4.

For this purpose, a cubic interpolation was used, and the mode shape of the plate was interpolated to decrease the sampling distance from $20 \mathrm{~mm}$ to $6.6 \mathrm{~mm}$. Furthermore, each mode shape was normalized.

\section{Analysis of measured signals}

The first mode shape of the stiffened plates was constructed by using the wavelet packet node norm. Fig. 20 displays the first mode shapes of experimental case one and simulated case one. Afterwards, 2-D DWT is used to analyze the first mode shape of the damaged plates. The results of case 1 (measurement area 1 and measurement area 2), case 2, case 3 and case 4 are shown in Fig. 21 to Fig. 25, respectively. By excluding the boundary condition's noising effect that occurred in case 1 (measurement area 2), the precision of locating the crack will not be affected in the other cases below. Nonetheless, there are still some noise signals in the experimental results.

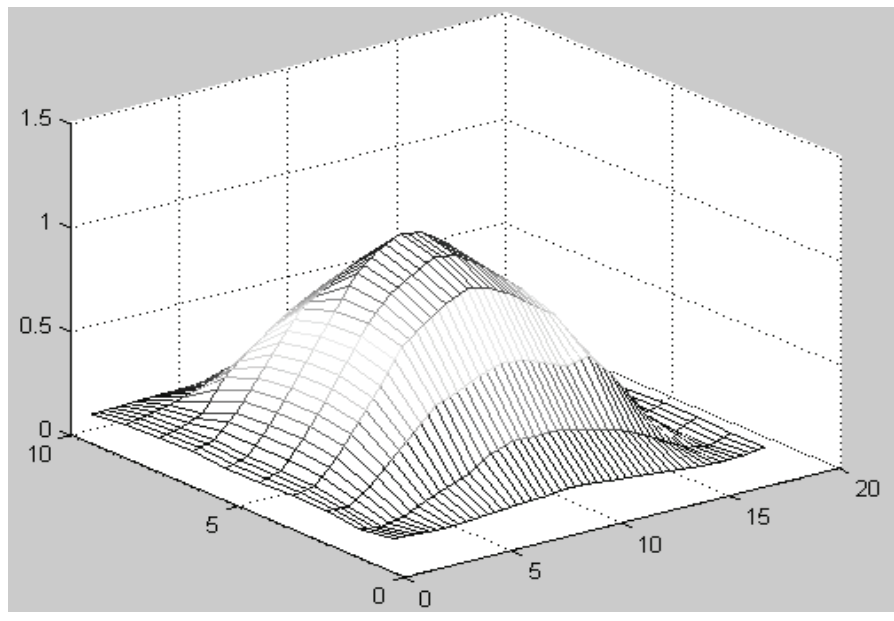

(a) expremental first mode shape.

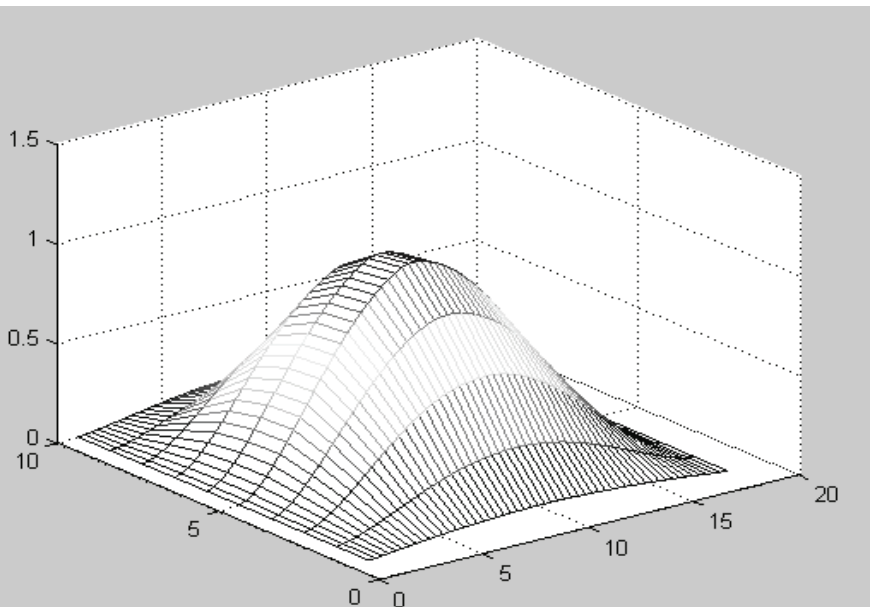

(b)simulated first mode shape.

Fig. 20 (a) The first mode shape of case1 obtained by using wavelet packet node norm and (b) First mode shape of case1 obtained by ANSYS(The scale in figure is $1: 3 \mathrm{~cm}$ ).

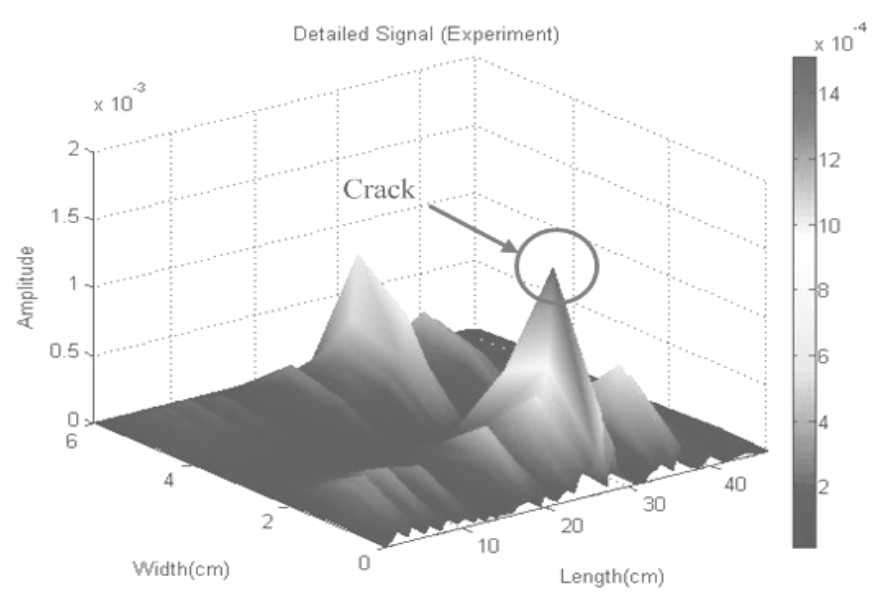

Fig. 21 Diagonal detailed signal of case1 (measurement area 1).

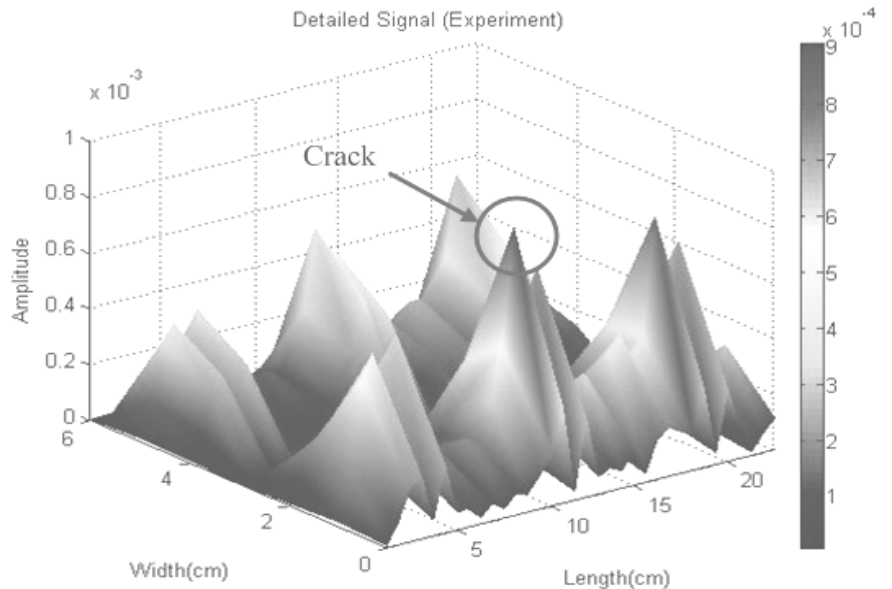

Fig. 22 Diagonal detailed signal of case1 (measurement area 2). 


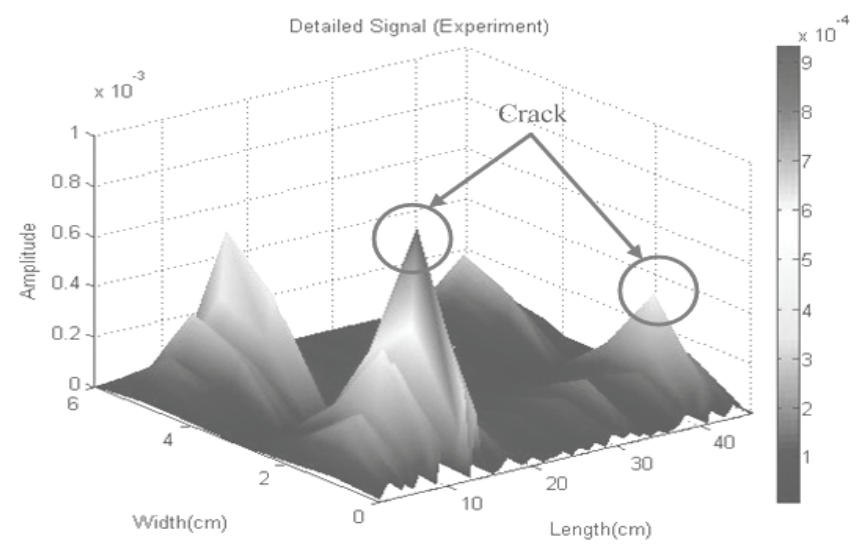

Fig. 23 Diagonal detailed signal of case 2.

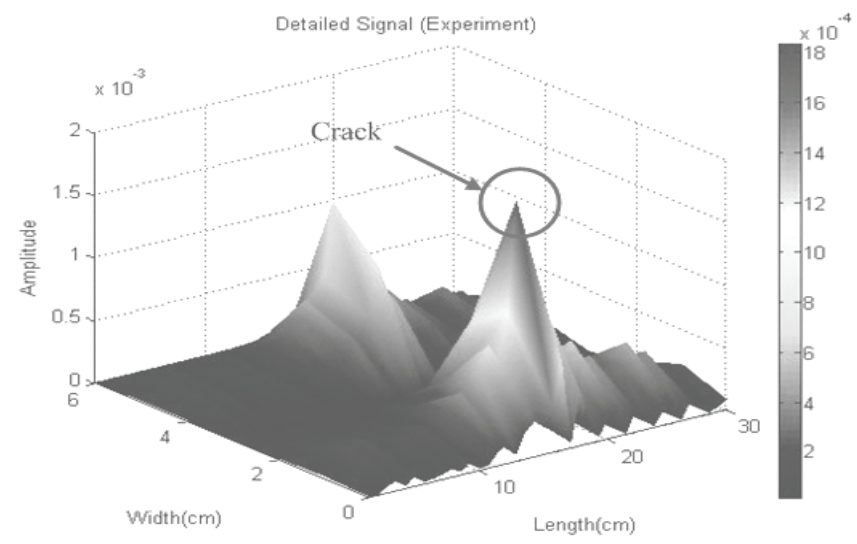

Fig. 24 Diagonal detailed signal of case 3

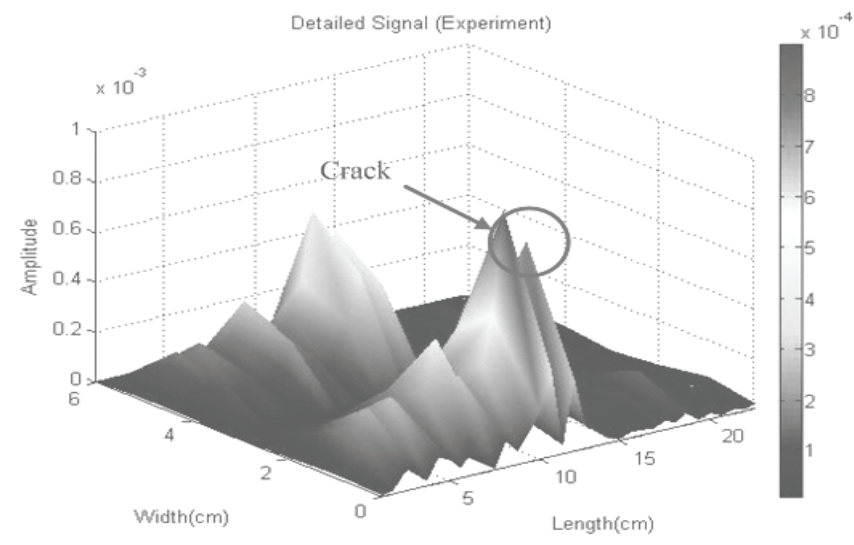

Fig. 25 Diagonal detailed signal of case 4.

\section{CONCLUSIONS}

The present investigation is primarily based on the numerical simulation and the experimental study to explore the active non-destructive damage detection method for stiffened plates by wavelet transform. Symlets 4 wavelet is appropriate for detecting the location of cracks in single and double-cracked stiffened plates. We can then determine the location of cracks through diagonal detailed signal. The advantage of the proposed technique is that the less expensive equipments are used to identify cracks or flaws in a stiffened plate. In addition, fewer measurement points are required by utilizing the proposed method in comparison to those presented in previous studies. Thus, the method may become a feasible non-destructive inspection practice for identification of small cracks of structural members as it also requires a shorter operation time compared to previous methods.

\section{ACKNOWLEDGEMENT}

This research was supported by the National Science Council, Taiwan, under the grants NSC 96-2221-E-006-323MY3.

\section{REFFERENCES}

Cawley, P. Adams, R.D., 1979. The Location of Defects in Structures from Measurements of Natural Frequencies. J Strain Analysis, 14, pp.309-313.

Daubechies, I., 1992. Ten Lectures on Wavelets. SIAM, Philadelphia.

Mallat, S.G., 1988. A Theory for Multiresolution Signal Decomposition: the Wave Representation. Comm. Pure Appl. Math, 41, pp. 674-693.

Meyer, Y., 1986. Ondettes et Functions Splines. Lectures given at the University of Torino, Italy.

Meyer, Y., 1986. Ondettes, Functions Splines et Analyses Graduees. Seminaire EDP, Ecole Polytechnique, Paris, France.

Morlet, J. Arens, G. Fourgeau, I. Giard, D., 1982. Wave Propagation and Sampling Theory. Geophysics, 47, pp. 203-236.

Morlet, J., 1983. Sampling Theory and Wave Propagation. NATO ASI Series, Issues in Acoustic Signal/Image Processing and Recognition, Vol. I, pp. 233-261.

P, Goupillaud. A, Grossman. J, Morlet., 1984. Cycle-Octave and Related Transforms in Seismic Signal Analysis. Geoexploration, Vol. 23, pp. 85-102.

Rucka, M. Wilde, K., 2006. Application of continuous wavelet transform in vibration based damage detection method for beams and plates. Journal of Sound and Vibration, 297, pp.536-550.

Surace, C. Ruotolo, R., 1994. Crack Detection of a Beam Using the Wavelet Transform. Proc. of the 12th International Modal Analysis Conference, Honolulu, pp.1141-1147.

Wang, W.J. McFadden, P.D., 1996. Application of Orthogonal Wavelets to Early Gear Damage Detection. Mechanical Systems and Signal Processing, 9, pp.497508.

Yang, J.M. Hwang, C.N. Yang, Yaubin., 2008. Crack identification of stiffened plates by two-dimensional discrete wavelet transform. International Conference of The Asian-Pacific Technical Exchange and Advisory Meeting on Marine Structures, Istanbul, Turkey. 